between response to $\mathrm{CS}+\mathrm{GH}$ and baseline protein levels $(\mathrm{p}=0,007)$ thus increasing the power of our prediction model (ROC up to 0,843 ). In the Celecoxib group, non parametric analysis showed increased levels only of TSP1 at baseline in responders compared to non-responders (R: $363,03 \pm 104,83 \mathrm{ng} / \mathrm{mL}$ vs NR: 331,95 $\pm 92,59 \mathrm{ng} / \mathrm{mL} ; \mathrm{p}=0,041)$, while no statistically significant differences were found for this protein in the $\mathrm{CS}+\mathrm{GH}$ group. When we include in the regression model 4 predictive variables ( 2 clinical and 2 analytical) and TSP1 as covariate, we found a specific interaction between response to Celecoxib and baseline protein levels $(p=0,045)$ thus increasing the predictive power of this model (ROC up to 0,749 ). Conclusions: Combining clinical and analytical parameters, we clinically validated (qualified) 2 panels of biomarkers that could efficiently predict OA patients response to $\mathrm{CS}+\mathrm{GH}$ with an accuracy of $84,3 \%$ or to Celecoxib with an accuracy of $74,9 \%$.

Disclosure of Interest: V. Calamia: None declared, F. Picchi: None declared, I. Rego: None declared, M. Camacho: None declared, L. González: None declared, P. Fernández-Puente: None declared, M. Herrero Employee of: Bioiberica S.A.U., H. Martínez Employee of: Bioiberica S.A.U., J. Vergés: None declared, C. RuizRomero: None declared, F. J. Blanco: None declared DOI: 10.1136/annrheumdis-2018-eular.3439

\section{SAT0585 DECREASED SYNOVIAL LEVELS OF DICKKOPF-1 ARE ASSOCIATED WITH RADIOLOGICAL PROGRESSION IN KNEE OSTEOARTHRITIS PATIENTS}

W.A. Hassan ${ }^{1}$, E.G. Behiry ${ }^{2} .{ }^{1}$ Rheumatology and Rehabilitation; ${ }^{2}$ Clinical and Chemical Pathology Department, Benha University Hospital, Benha, Egypt

Background: Dickkopf-1 (Dkk-1) is a direct inhibitory ligand of Wnt $\beta$-catenin signalling pathway that act through binding to low-density lipoprotein (LDL) related proteins (LRP5/6) receptors.Dkk-1 is considered an important mediator of cartilage homeostasis and skeletal remodelling. ${ }^{1}$

Objectives: This study aimed to measure serum and synovial fluid (SF) levels of Dkk-1 in patients with early primary knee osteoarthritis (KOA) and to examine the relationship between these levels and the clinical and functional parameters as well as radiological progression of KOA

Methods: We measured Dkk-1 in the serum $(n=48)$ and SF samples $(n=22)$ from 48 early KOA patients and in the serum from healthy control $(n=30)$. Body mass index (BMI), numerical rating scale of pain (NRSP) and The Western Ontario Mc Master scale (WOMAC) were recorded. Plain radiographs using Osteoarthritis Research Society International (OARSI) atlas to assess joint space narrowing $(\mathrm{JSN})^{2}$ and musculoskeletal ultrasound examination (MSUS) were performed at baseline and after 24 months to assess radiological progression. Radiological progression was considered if there is more than 2 points increase in JSN score or transition to higher grade in MSUS examination ${ }^{3}$ at the 24 months follow up period compared to baseline grade.

Results: SF Dkk-1 levels were significantly decreased (mean \pm SD 115.05 $\pm 34.2 \mathrm{pg} / \mathrm{ml}$ ) compared to their paired serum levels (mean \pm SD 969.82 $\pm 365.72 \mathrm{pg} / \mathrm{ml}$ ), $\mathrm{p}<0.001$ in KOA patients. There was no significant difference in serum Dkk-1 levels between KOA patients and healthy controls (mean \pm SD $988.77 \pm 385.19 \mathrm{pg} / \mathrm{ml}$ and $1084.73 \pm 408.38 \mathrm{pg} / \mathrm{ml}$ respectively), the SF concentrations of Dkk-1 significantly correlated with the baseline thickness of the cartilage on the medial condyle $(0.53, \mathrm{p}<0.05)$ but not on the lateral condyle of the femur $(r=0.11, p>0.05)$.there was no significant correlation between serum Dkk-1and baseline cartilage thickness on medial and lateral condyles $((r=-0.13$ and -0.09 respectively, $p<0.05)$. Patients in the least quartile of SF Dkk-1had an increased risk of radiological progression with plain radiography and MSUS (age, sex and $\mathrm{BMI}$ adjusted RR 2.1 and $3.4,95 \% \mathrm{CI}$ respectively.

Conclusions: Osteoarthritis patients have significantly lower synovial levels than serum levels of Dickkopf-1 that were obviously associated with radiological progression on plain radiography and MSUS suggesting that it could be a useful marker to reflect OA severity and implies a possible role in the pathogenesis of $\mathrm{OA}$.

\section{REFERENCES:}

[1] Min S, Wang C, Lu W, Xu Z, Shi D,Chen D, et al. Serum levels of the bone turnover markers dickkopf-1, osteoprotegerin, and TNF- $\alpha$ in knee osteoarthritis patients. Clin Rheumatol 2017 Oct;36(10):2351-2358.

[2] Altman RD, Hochberg M, Murphy WA, Jr, Wolfe F, Lequesne M. Atlas of individual radiographic features in osteoarthritis. Osteoarthritis Cartilage 1995;3(Suppl A):3-70.

[3] Saarakkala S, Waris P, Waris V, Tarkiainen I, Karvanen E, Aarnio J, et al. Diagnostic performance of knee ultrasonography for detecting degenerative changes of articular cartilage. Osteoarthritis Cartilage 2012;20:376-81.

Disclosure of Interest: None declared

DOI: 10.1136/annrheumdis-2018-eular.4608

\section{SAT0586 \\ RESULTS FROM A 52 WEEK RANDOMISED, DOUBLE- BLIND, PLACEBO-CONTROLLED, PHASE 2 STUDY OF A NOVEL, WNT PATHWAY INHIBITOR (SM04690) FOR KNEE OSTEOARTHRITIS TREATMENT}

Y. Yazici ${ }^{1}$, T. McAlindon ${ }^{2}$, A. Gibofsky ${ }^{3}$, N. Lane ${ }^{4}$, D. Clauw ${ }^{5}$, C. Swearingen ${ }^{1}$, A. DiFrancesco ${ }^{1}$, J. Tambiah", M. Hochberg $6 .{ }^{1}$ Samumed, LLC, San Diego; ${ }^{2}$ Tufts Medical Center, Boston; ${ }^{3}$ Hospital for Special Surgery, New York; ${ }^{4}$ UC Davis Medical Center, Davis: ${ }^{5}$ University of Michigan Medical School, Ann Arbor,

${ }^{6}$ University of Maryland School of Medicine, Baltimore, USA

Background: Knee osteoarthritis $(\mathrm{OA})$ is characterised by pain, disability and joint deformity due to articular cartilage degradation and bone remodelling. Wnt signalling is involved in these cellular processes. SM04690, a small molecule Wnt pathway inhibitor, is in development as a potential disease modifying OA drug (DMOAD) for knee OA.

Objectives: A phase 2, multicenter, 52 week, placebo-controlled (PBO) trial was conducted to identify a target population, determine optimal dose and assess safety.

Methods: Knee OA subjects, Kellgren-Lawrence $(\mathrm{KL})$ grades 2-3, received a sin gle $2 \mathrm{~mL}$ injection of SM04690 $0.03 \mathrm{mg}, 0.07 \mathrm{mg}, 0.23 \mathrm{mg}$ or PBO in target (most painful) knee. WOMAC Pain and Function were assessed (Weeks 0, 4, 13, 26 39,52 ) and fixed flexion radiographs (QuAP positioned; Weeks 0, 26, 52) assessed medial joint space width (mJSW). Analysis of covariance adjusted for baseline was conducted using multiple imputation for missing data. Exploratory subgroups included: 1) unilateral symptomatic subjects (pre-specified; determined by history and examination) and 2) unilateral symptomatic subjects withou comorbid pain (post-hoc; Widespread Pain Index $\leq 4$, Symptom Severity $\leq 2$ [WP-]). Results: 455 subjects (mean age 60.3 [ \pm 8.7$]$ years, BMI $29.9[ \pm 4.6] \mathrm{kg} / \mathrm{m}^{2}$, female $58.9 \%$, KL grade 3 [64.4\%], unilateral symptomatic OA [36.0\%]) were enrolled, $91 \%$ with radiographic bilateral OA. Seventeen serious adverse events, all unrelated to SM04690, were reported.

The primary endpoint of Week 13 change from baseline in WOMAC Pain was not met. In ITT, at all timepoints, minimal clinically important differences ( $>10 \%$ full range) in WOMAC Pain and Function compared to baseline were seen in all groups. In $0.07 \mathrm{mg}$ unilateral symptomatic subjects, at 52 weeks, WOMAC Pain (4.4, $\mathrm{p}=0.049)$, and Function $(17.5, \mathrm{p}=0.035)$ were significantly improved compared to PBO. In $0.07 \mathrm{mg}$ unilateral symptomatic WP-subjects at Weeks 26, 39 , and 52, WOMAC Pain (4.6, $\mathrm{p}=0.039 ; 5.9, \mathrm{p}=0.042$; and $5.6, \mathrm{p}=0.025$, respectively) and Function (16.3, $p=0.027 ; 19.7, p=0.035$; and 22.8, $p=0.017$, respec tively) were significantly improved compared to PBO (Abstract SAT0586 - figure 1).

At 26 and 52 weeks, $0.07 \mathrm{mg}$ unilateral symptomatic $(0.5 \mathrm{~mm}, \mathrm{p}=0.006$ and $0.4 \mathrm{~mm}, \mathrm{p}=0.021$, respectively) and $0.07 \mathrm{mg}$ unilateral symptomatic WP ( $0.5 \mathrm{~mm}, p=0.016$ and $0.4 \mathrm{~mm}, p=0.032$, respectively) subgroups demonstrated significant increases from baseline in mJSW compared to PBO (figure 1).
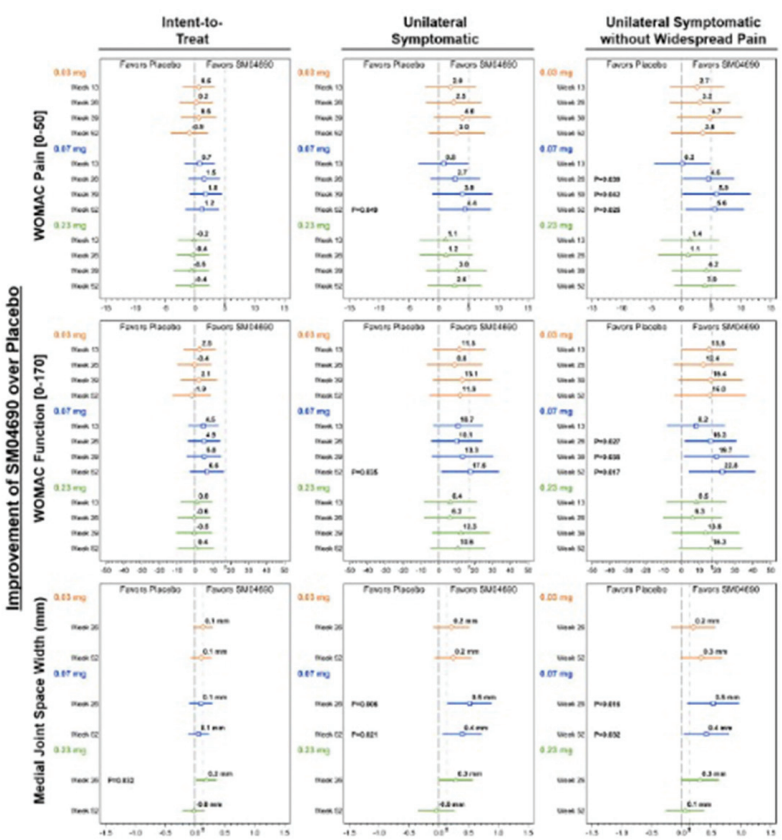

Abstract SAT0586 - Figure 1. Ladder plots depicting mean improvement (and 95\% confidence intervals) of SMO4690 over placebo adjusted for baseline. 